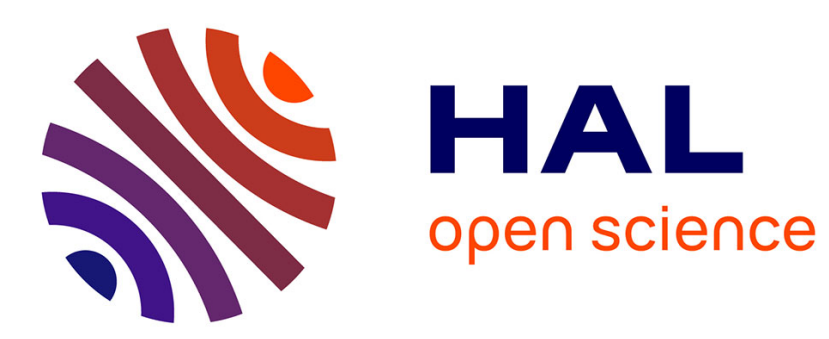

\title{
A semi-numerical model for near-critical angle scattering
} L.J. Fradkin, M. Darmon, S. Chatillon, P. Calmon

\section{To cite this version:}

L.J. Fradkin, M. Darmon, S. Chatillon, P. Calmon. A semi-numerical model for near-critical angle scattering. Journal of the Acoustical Society of America, 2016, 139 (1), pp.141-150. $10.1121 / 1.4939494$. cea-01845384

\section{HAL Id: cea-01845384 https://hal-cea.archives-ouvertes.fr/cea-01845384}

Submitted on 9 Jan 2019

HAL is a multi-disciplinary open access archive for the deposit and dissemination of scientific research documents, whether they are published or not. The documents may come from teaching and research institutions in France or abroad, or from public or private research centers.
L'archive ouverte pluridisciplinaire HAL, est destinée au dépôt et à la diffusion de documents scientifiques de niveau recherche, publiés ou non, émanant des établissements d'enseignement et de recherche français ou étrangers, des laboratoires publics ou privés. 


\title{
A semi-numerical model for near-critical angle scattering
}

\author{
Larissa Ju. Fradkin ${ }^{a}$ \\ Sound Mathematics Ltd., Cambridge CB4 2AS, United Kingdom \\ Michel Darmon, Sylvain Chatillon, and Pierre Calmon \\ Department of Imaging and Simulation for NDT (Non-Destructive Testing), CEA (Commissariat à l'énergie \\ atomique et aux énergies alternatives), LIST (Laboratoire d'Intégration de Systèmes et des technologies), \\ F-91191 Gif-sur-Yvette, France
}

\begin{abstract}
Numerous phenomena in the fields of physics and mathematics as seemingly different as seismol-ogy, ultrasonics, crystallography, photonics, relativistic quantum mechanics, and analytical number theory are described by integrals with oscillating integrands that contain three coalescing critical-ities, a branch point, stationary phase point, and pole as well as accumulation points at which the speed of integrand oscillation is infinite. Evaluating such integrals is a challenge addressed in this paper. A fast and efficient numerical scheme based on the regularized composite Simpson's rule is proposed, and its efficacy is demonstrated by revisiting the scattering of an elastic plane wave by a stress-free half-plane crack embedded in an isotropic and homogeneous solid. In this canonical problem, the head wave, edge diffracted wave, and reflected (or compensating) wave each can be viewed as a respective contribution of a branch point, stationary phase point, and pole. The pro-posed scheme allows for a description of the non-classical diffraction effects near the "critical" rays (rays that separate regions irradiated by the head waves from their respective shadow zones). The effects include the spikes present in diffraction coefficients at the critical angles in the far field as well as related interference ripples in the near field.
\end{abstract}

\section{INTRODUCTION}

Wave scattering can be described using integral equations, which involve integration over scattering surfaces. There are many exact numerical schemes for solving them; however, in the far field, otherwise known as the highfrequency or small wavelength regime, these are difficult to implement, and approximations prove more useful. In this regime the corresponding integrands are products of rapidly oscillating exponents and slowly varying amplitudes. This means that the scattering integrals can be evaluated asymptotically, with the contributions coming from isolated or coalescing criticalities, that is, poles, stationary phase points, and branch points. In physical terms, contributions from isolated criticalities describe the geometrical regions supporting, respectively, GO/GA/GE (geometrical optics/acoustics/elastodynamics) waves, edge diffracted waves, and head waves, while contributions from coalescing criticalities describe transition zones in between. ${ }^{1}$

The most popular approximate theories are KA (the Kirchhoff approximation) $)^{2,3}$ and GTD (Geometrical Theory of Diffraction), ${ }^{4,5}$ both applicable where wave fronts and scattering surfaces are locally plane and scatterers' edges are locally straight. In the shadow both postulate the existence of the field compensating the incident field. KA involves an additional assumption that the scattered field on the irradiated/shadow side of the scatterer is simply the reflected/ compensating field, reducing the problem to evaluation of a

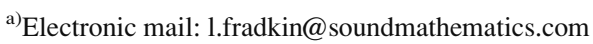

surface integral. Mathematically speaking, the GO/GA/GE reflected waves and compensating fields are contributions of the isolated poles in the corresponding integrands.

In the electromagnetic and acoustic GTD, each ray incident on a sharp edge and carrying a high-frequency wave produces one cone of diffracted rays. ${ }^{4}$ In an isotropic solid two cones are generated, with the rays forming the surfaces of the inner and outer cone, which carry the longitudinal and transverse motions, respectively. The half-angles of the cones are determined by the Snell law of diffraction. KA estimates the amplitudes of the diffracted waves generated by the straight edge too, but at smaller angles to the edge GTD provides a much better recipe. GTD is valid only in the geometrical regions where the diffracted waves do not interfere with other waves. Mathematically speaking, the diffracted waves are asymptotic contributions of the isolated stationary phase points in the corresponding integrands. The GTD amplitudes carried by each diffracted ray decrease as the square root of both distance to the tip of the diffraction cone and wave frequency and involve edge diffraction coefficients dependent on directions of the incident and diffracted rays only. GTD is used in radar technology, design of reflector antennas, and ultrasonic NDE (Non-Destructive Evaluation of industrial materials). ${ }^{6-8}$

PTD (Physical Theory of Diffraction) is the theory that extends the classical KA using the exact description of edge diffracted waves. ${ }^{2,3}$ In the high-frequency regime, when such a description is unknown the corrections to the Kirchhoff diffraction coefficients can be obtained using their GTD counterparts. ${ }^{2,3,9}$ Unlike GTD and KA diffraction 
coefficients, the corrections have no singularities at the shadow boundaries. The high-frequency PTD leads to an extension of GTD to "penumbras" (transition zones between regions irradiated by $\mathrm{GO} / \mathrm{GA} / \mathrm{GE}$ waves and their respective shadow zones). Mathematically speaking, the total penumbral field is the contribution of a coalescing pole and stationary phase point.

Neither KA nor GTD nor high-frequency PTD is valid on the "critical" rays (rays that separate regions irradiated by the head waves from their respective shadow zones) or critical zones (the transition zones that surround critical rays)see Fig. 1, where, for simplicity of presentation, the incident ray is normal to the crack, so that both diffraction cones degenerate into cylindrical surfaces; the shaded regions are the corresponding critical zones. Mathematically speaking, the head wave is an asymptotic contribution of an isolated branch point and the fields in the critical zones are the contributions of the coalescing stationary phase point and branch point. The head waves in Fig. 1 have conical fronts. They travel along the boundary with the longitudinal speed $c_{L}$, detach from it at the critical angle, and propagate in the solid with the transverse speed $c_{T}<c_{L}$. Waves of this nature were first discovered in geophysics ${ }^{10,11}$ and are a manifestation of an ubiquitous phenomenon of total internal refection.

No high-frequency asymptotics are available when the critical transition zones overlap penumbras, ${ }^{12,13}$ that is when all three critical points coalesce, but when they do not the critical zones can be described using asymptotics involving the Weber functions or their variant, the parabolic cylinder functions. $^{1,11,12,14}$ In some situations of practical interest none of these asymptotics prove satisfactory. ${ }^{15}$ The need to resolve this difficulty has been the main rationale for the present study.

Our aim is threefold: First, a novel fast and efficient numerical scheme is proposed for evaluating integrals with oscillating integrands that contain three coalescing criticalities, a branch point, stationary phase point, and pole. Such integrals arise not only in scattering ${ }^{5}$ but also in

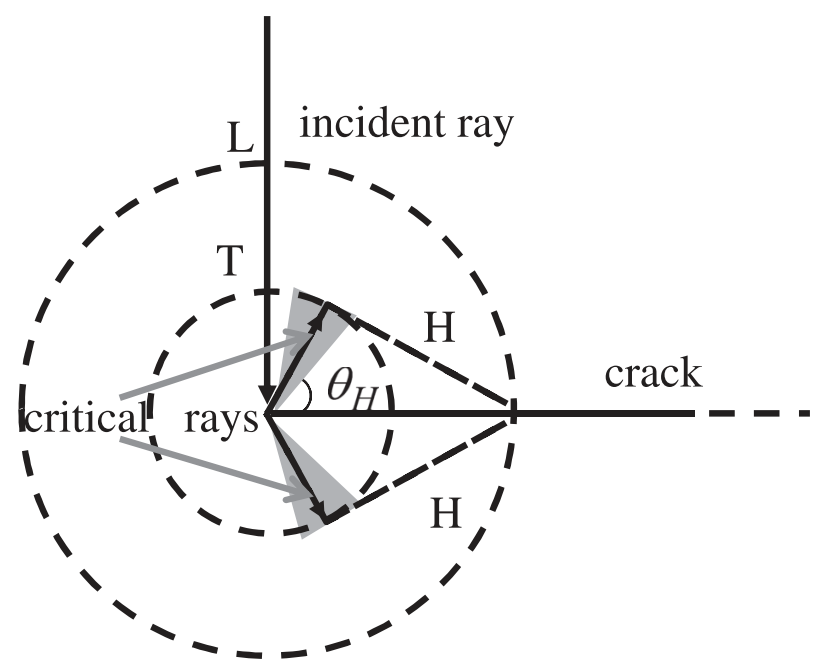

FIG. 1. Representative edge diffracted rays and wave curves (cross-sections of wave fronts). The key: $L$-longitudinal wave, $T$-transverse wave, $H$ head wave; dashed lines-wave curves, solid lines-critical rays, shaded regions-critical zones. crystallography, ${ }^{16}$ photonics, ${ }^{17}$ relativistic quantum mechanics, ${ }^{18}$ and even analytic number theory. ${ }^{19}$ Our scheme deals with an additional complication-the presence in the integration domain of "accumulation" points (the points at which the speed of oscillation is infinite), which are also weak singularities. Second, efficacy of the proposed scheme is demonstrated by revisiting the canonical problem of scattering of a transverse plane harmonic wave by a stress-free half-plane crack in an isotropic homogeneous solid. This problem has been chosen to test the scheme, because it has a well-known analytical solution ${ }^{5}$ and because PTD can utilize this solution to simulate complex large scatterers. Third, hybrid schemes are introduced relying on the proposed numerical scheme, if the branch and phase stationary points coalesce, and on the classical GTD or high-frequency PTD, otherwise.

The paper is organized as follows: In Sec. II the canonical problem of scattering of a harmonic plane wave by a stress-free half-plane crack in a homogeneous and isotropic solid is stated. In Sec. III the well-known exact solution of this problem ${ }^{21}$ is given in an integral form, and in Sec. IV the GTD and high-frequency PTD asymptotics of this solution are described. In Sec. V this exact solution is rewritten in the form of an integral scattering coefficient, and a novel numerical scheme is offered for its evaluation. Section VI is devoted to cross-validation of this scheme and GTD and high-frequency PTD. In Sec. VII the scheme is incorporated into a semi-numerical schedule applicable at any distance to diffracting edge. Conclusions are presented in Sec. VIII.

\section{STATEMENT OF THE CANONICAL EDGE DIFFRACTION PROBLEM}

The following conventions are used throughout: When the same symbol appears in bold and regular font, the bold font is used to denote a vector quantity and the regular font, its amplitude. Whenever appropriate, the field symbols are supplied with superscripts and subscripts describing the nature of the motions. In particular, the descriptors inc, tot, sc, $\mathrm{GE}$, and d denote, respectively, the incident, total, scattered, geometrico-elastodynamic, and diffracted waves. The descriptors $\alpha, \beta=\mathrm{L}$, TH, TV, or Ray refer to the longitudinal, transverse horizontal, transverse vertical, or Rayleigh wave, respectively, with superscript $\alpha$ reserved for the incident modes and subscript $\beta$ for the scattered ones. Numerical subscripts are used to refer to base vectors or else the corresponding vector components. Everywhere $c$ is wave speed and wavenumber $k=\omega / c$, with $\omega$ the circular frequency.

The three-dimensional space is assumed to be filled with an isotropic homogeneous solid. In the presence of a scatterer, $\mathbf{U}^{\text {tot }}$, the total displacement field in this solid is the sum of incident field $\mathbf{U}^{\text {inc }}$ and scattered field $\mathbf{U}^{\text {sc }}$. In the high frequency approximation, the field scattered by a scatterer with an edge is the sum of the geometrico-elastodynamic field $\mathbf{U}^{\mathrm{GE}}$, classical diffracted field $\mathbf{U}^{\mathrm{d}}$, the surface Rayleigh wave $\mathbf{U}^{\text {Ray }}$, and head wave $\mathbf{U}^{\mathrm{H}}$.

Let the scatterer be a semi-infinite planar crack, and the Cartesian coordinate system be chosen so that the positive $\mathbf{e}_{1}$-axis lies on the crack and the $\mathbf{e}_{2}$-axis runs along the crack 
edge (see Fig. 2). Then every vector $\mathbf{v}$ can be described by the corresponding components $\left(v_{1}, v_{2}, v_{3}\right)$. Below only timeharmonic waves $\boldsymbol{U}(\boldsymbol{x}, t)=\boldsymbol{u}(\boldsymbol{x}) \exp (-i \omega t)$ are considered, with $i$ the imaginary unity; $\boldsymbol{x}=\left(x_{1}, x_{2}, x_{3}\right)$ an observation point; $t$ is the time; and the factor $\exp (-i \omega t)$ is implied but omitted everywhere. The equation of motion is

$$
\nabla \cdot \sigma^{\mathrm{tot}}(\boldsymbol{x})=\rho \frac{\partial^{2} \boldsymbol{u}^{\mathrm{tot}}}{\partial t^{2}}(\boldsymbol{x})
$$

where $\nabla=\left(\partial / \partial x_{1}, \partial / \partial x_{2}, \partial / \partial x_{3}\right)$ is the nabla operator; $\sigma$ is the stress tensor; ${ }^{5}$ and $\rho$ is the solid density. The crack faces are assumed to be stress-free,

$$
\left.\boldsymbol{t}^{\mathrm{tot}}(\boldsymbol{x})\right|_{\text {crack }}=\mathbf{0},
$$

where the traction $\boldsymbol{t}\left(\boldsymbol{x}^{ \pm}\right)= \pm \boldsymbol{e}_{3} \cdot \sigma(\boldsymbol{x})$ and $\mathbf{x}^{+}$and $\mathbf{x}^{-}$are points on the irradiated and shadow side of the crack, respectively. ${ }^{5}$ It is further assumed that the scattered waves satisfy the radiation condition at infinity, which demands that they are outgoing. ${ }^{5,21}$

Using the Green's stress tensor $\sigma^{G}$ and crack opening displacement $\Delta \boldsymbol{u}^{\alpha(\mathrm{sc})}(\boldsymbol{x})=\boldsymbol{u}^{\alpha(\mathrm{sc})}\left(\boldsymbol{x}^{+}\right)-\boldsymbol{u}^{\alpha(\mathrm{sc})}\left(\boldsymbol{x}^{-}\right)$this problem can be reformulated as the integral equation ${ }^{5}$

$$
\boldsymbol{u}^{\alpha(\mathrm{sc})}(\boldsymbol{x})=\frac{k_{L}}{2 \pi} \int_{0}^{\infty} d x_{1}^{\prime} \int_{-\infty}^{\infty}\left(\mathbf{e}_{3} \cdot \sigma^{G}\right)\left(\boldsymbol{x}-\boldsymbol{x}^{\prime}\right) \Delta \boldsymbol{u}^{\alpha(\mathrm{sc})}\left(\boldsymbol{x}^{\prime}\right) d x_{2}^{\prime} .
$$

\section{THE ANALYTICAL SOLUTION OF THE CANONICAL EDGE DIFFRACTION PROBLEM}

Solving the above problem confirms that isotropic media support $L$ (longitudinal) and $T$ (transverse) plane waves $\boldsymbol{u}=A \boldsymbol{d} \exp (i k \boldsymbol{p} \cdot \boldsymbol{x})$. Any polarization vector $\boldsymbol{d}^{L}\left(\boldsymbol{d}^{T}\right)$ is parallel (perpendicular) to propagation vector $\boldsymbol{p}$. Any $\boldsymbol{d}^{T}$ is traditionally decomposed with respect to a reference plane into a vertical component $T V$ and a horizontal component $T H$. The reference plane can be the plane of the crack $^{21}$ or incidence plane. ${ }^{5,20}$

Defining the single Fourier transform of any integrable function $g\left(x_{1}\right)$ to be

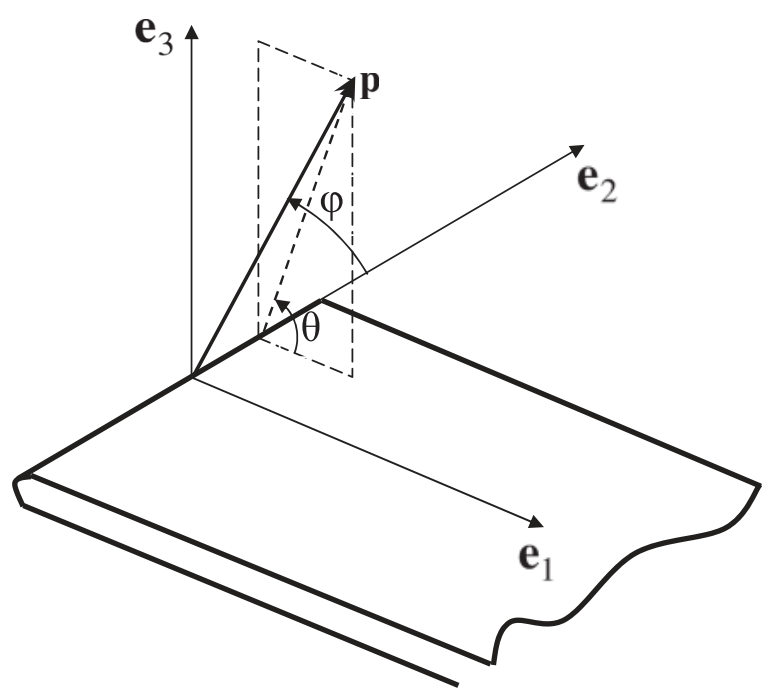

FIG. 2. The coordinate systems associated with the crack.

$$
\hat{g}\left(\xi_{1}\right)=\frac{k_{L}}{2 \pi} \int_{-\infty}^{\infty} g\left(x_{1}\right) \mathrm{e}^{-i k_{L} \xi_{1} x_{1}} d x_{1}
$$

it has been shown ${ }^{21}$ that the scattered field is the inverse Fourier transform

$$
\boldsymbol{u}^{\alpha(\mathrm{sc})}(\boldsymbol{x})=\int_{-\infty}^{\infty} \hat{\boldsymbol{u}}^{\alpha(\mathrm{sc})}\left(\xi_{1}, x_{2}, x_{3}\right) \mathrm{e}^{i k_{L} \xi_{1} x_{1}} d \xi_{1},
$$

with $\hat{\boldsymbol{u}}^{\alpha(\mathrm{sc})}\left(\xi_{1}, x_{2}, x_{3}\right)$ being the sum of all scattered modes,

$$
\begin{aligned}
\hat{\boldsymbol{u}}^{\alpha(\mathrm{sc})}\left(\xi_{1}, x_{2}, x_{3}\right)= & \frac{-i}{\kappa_{T}^{2}\left(\xi_{1}+\xi_{1}^{\alpha}\right)} \sum_{\beta=L, T V, T H} \frac{\kappa_{\beta}^{2}}{\gamma_{\beta}\left(\xi_{1}\right)} \\
& \times\left[\boldsymbol{v}_{\beta}(\zeta) \cdot \boldsymbol{v}^{\alpha}\left(\zeta^{\alpha}\right)\right] \boldsymbol{d}_{\beta}(\zeta) \mathrm{e}^{i k_{L}\left[\xi_{2} x_{2}+\gamma_{\beta}\left(\xi_{1}\right)\left|x_{3}\right|\right]} .
\end{aligned}
$$

Above $\kappa_{L}=1 ; \kappa_{T}=c_{L} / c_{T}>1 ; \zeta=\left(-\xi_{1}, \xi_{2}, s \gamma_{\beta}\left(\xi_{1}\right)\right)=$ $\kappa \boldsymbol{p}$ is a dimensionless wave vector; and $s=\operatorname{sgn}\left(x_{3}\right)$, with $\gamma_{\beta}\left(\xi_{1}\right)=\sqrt{\kappa_{\beta}^{2}-\xi_{1}^{2}-\xi_{2}^{2}}$. There is no need to reproduce here the expressions for displacement vectors $\boldsymbol{d}$ and vectors $\boldsymbol{v}$, only to note that for $\beta=T(T V$ or $T H)$ all third components $v_{T, 3}(\zeta)$ contain a factor $\sqrt{\xi_{1}+\sqrt{1-\xi_{2}^{2}}}$ and thus the branch point $\xi_{\text {br }}=-\gamma_{L}(0)$. Also, each component of each $T$-integrand contains pole $\xi_{\mathrm{p}}=-\xi_{1}^{\alpha}$ and has phase $k_{L} \zeta_{T} \cdot \mathbf{x}=$ $k_{L}\left(-\xi_{1} x_{1}+\xi_{2} x_{2}+\gamma_{T}\left(\xi_{1}\right)\left|x_{3}\right|\right)$. The stationary phase point $\xi_{s t}$ is denoted by $-\xi_{T, 1}$. Since the problem is invariant with respect to translations along the $\boldsymbol{e}_{2}$-direction all dimensionless wave vectors $\zeta$ have the same second component $\xi_{2}$.

\section{THE FAR FIELD APPROXIMATIONS FOR THE CANONICAL EDGE DIFFRACTION PROBLEM}

In order to find the KA to the Green's formula (3), which is based on Eq. (5), it is helpful to note that under this approximation, on the crack surface, the single Fourier transform of the scattered field is the sum of GE modes ${ }^{5}$

$$
\begin{aligned}
\hat{\boldsymbol{u}}^{\alpha(\mathrm{GE})}\left(\xi_{1}, x_{2}, 0\right)= & \frac{-i}{\kappa_{T}^{2}\left(\xi_{1}+\xi_{1}^{\alpha}\right)} \sum_{\beta=L, T V, T H} \frac{\kappa_{\beta}^{2}}{\gamma_{\beta}\left(\xi_{1}\right)} \\
& \times\left[\boldsymbol{v}_{\beta}\left(\zeta_{\beta}^{\mathrm{GE}}\right) \cdot \boldsymbol{v}^{\alpha}\left(\boldsymbol{\zeta}^{\alpha}\right)\right] \boldsymbol{d}_{\beta}\left(\boldsymbol{\zeta}_{\beta}^{\mathrm{GE}}\right) \mathrm{e}^{i k_{L} \xi_{2} x_{2}},
\end{aligned}
$$

with $\zeta_{\beta}^{\mathrm{GE}}=\left(-\xi_{1}^{\alpha}, \xi_{2}, s \gamma_{\beta}\left(\xi_{1}^{\alpha}\right)\right)$. Therefore, using Eqs. (5) and (6) the KA scattered field is

$$
\begin{aligned}
\boldsymbol{u}^{\alpha(\mathrm{Kir})}(\boldsymbol{x})= & -\frac{i}{\kappa_{T}^{2}} \sum_{\beta=\mathrm{L}, \mathrm{TV}, \mathrm{TH}}\left[\boldsymbol{v}_{\beta}\left(\boldsymbol{\zeta}_{\beta}^{\mathrm{GE}}\right) \cdot \boldsymbol{v}^{\alpha}\left(\boldsymbol{\zeta}^{\alpha}\right)\right] \\
& \times \boldsymbol{d}_{\beta}\left(\boldsymbol{\zeta}_{\beta}^{\mathrm{GE}}\right) \int_{-\infty}^{\infty} \frac{\kappa_{\beta}^{2}}{\gamma_{\beta}\left(\xi_{1}\right)} \frac{\mathrm{e}^{i k_{L} \zeta_{\beta} \cdot \mathbf{x}}}{\xi_{1}+\xi_{1}^{\alpha}} d \xi_{1} .
\end{aligned}
$$

Both integrals (5) and (8) can be evaluated in the far field approximation $k_{L} \sqrt{x_{1}^{2}+x_{2}^{2}+x_{3}^{2}} \gg 1$. In order to describe the result in a traditional form we introduce (see Fig. 2) the Cartesian coordinate system with the origin at the 
diffraction point $\boldsymbol{x}_{\beta}=\left(0, x_{\beta, 2}, 0\right)$ on the edge and the zenith direction $\mathbf{e}_{2}$, with the corresponding polar angle $\phi$ and azimuthal angle $\theta$. Then the edge diffracted field, the contribution to integral (5) of the isolated stationary point $\xi_{\mathrm{st}}$ is ${ }^{21}$

$$
\boldsymbol{u}_{\beta}^{\alpha(d)}(\boldsymbol{x}) \simeq \boldsymbol{D}_{\beta}^{\alpha(\mathrm{GTD})}\left(\theta_{\beta}, \phi^{\alpha}, \theta^{\alpha}\right) \frac{\mathrm{e}^{i k_{L}\left[\zeta^{\alpha} \cdot \boldsymbol{x}_{\beta}+\kappa_{\beta} R_{\beta}\right]}}{\left(k_{\beta} R_{\beta}\right)^{1 / 2}},
$$

where the scalar diffraction coefficient is ${ }^{21}$

$$
D_{\beta}^{\alpha(\mathrm{GTD})}\left(\theta_{\beta}, \phi^{\alpha}, \theta^{\alpha}\right)=\frac{\kappa_{\beta}^{3} \mathrm{e}^{i \pi / 4}}{\sqrt{2 \pi} \kappa_{T}^{2} \gamma_{\beta}(0)} \frac{\boldsymbol{v}_{\beta}\left(\boldsymbol{\zeta}_{\beta}\right) \cdot \boldsymbol{v}^{\alpha}\left(\boldsymbol{\zeta}^{\alpha}\right)}{\xi_{\beta, 1}-\xi_{1}^{\alpha}},
$$

$i k_{L} \zeta^{\alpha} \cdot x_{\beta}$ is the phase of the incident wave at the diffraction point $\boldsymbol{x}_{\beta}$; the distance from $\boldsymbol{x}_{\beta}$ to observation point $R_{\beta}=\sqrt{x_{1}^{2}+\left(x_{2}-x_{\beta, 2}\right)^{2}+x_{3}^{2}}$; and its radius-vector is

$$
\begin{aligned}
\boldsymbol{x}-\boldsymbol{x}_{\beta} & =\kappa_{\beta}^{-1} R_{\beta} \zeta_{\beta} \\
& =R_{\beta}\left(-\sin \phi_{\beta} \cos \theta_{\beta}, \cos \phi_{\beta}, \sin \phi_{\beta} \sin \theta_{\beta}\right),
\end{aligned}
$$

implying that $x_{\beta, 2}=x_{2}-R_{\beta} \cos \phi_{\beta}$ and $x_{j}=\xi_{\beta, j} R_{\beta} / \kappa_{\beta}$, $j=1,3$. Note that each vector diffraction coefficient $\boldsymbol{D}=\boldsymbol{D} \boldsymbol{d}$. Note too that given an incident angle $\phi^{\alpha}$ and observation point $\mathbf{x}$, the Snell law of diffraction $\kappa_{\beta} \cos \phi_{\beta}$ $=\kappa_{\alpha} \cos \phi^{\alpha}$ allows one to find the half solid angles $\phi_{\beta}$ of both diffraction cones and therefore to find both $\boldsymbol{x}_{\beta}$ 's.

Analogously, the Kirchhoff diffracted field can be found as the contribution to the integral [Eq. (8)] of the isolated stationary point $-\xi_{\beta, 1}$. It has the same form as Eq. (9) but with the GTD diffraction coefficient replaced by the Kirchhoff diffraction coefficient,

$$
D_{\beta}^{\alpha(\mathrm{Kir})}\left(\theta_{\beta}, \phi^{\alpha}, \theta^{\alpha}\right)=\frac{\kappa_{\beta}^{3} \mathrm{e}^{i \pi / 4}}{\sqrt{2 \pi} \kappa_{T}^{2} \gamma_{\beta}(0)} \frac{\boldsymbol{v}_{\beta}\left(\zeta_{\beta}^{\mathrm{GE}}\right) \cdot \boldsymbol{v}^{\alpha}\left(\zeta^{\alpha}\right)}{\xi_{\beta, 1}-\xi_{1}^{\alpha}} .
$$

As mentioned in Sec. I, outside the critical zones the scattered field can be described by using the high-frequency version of PTD, which has no unphysical singularities in penumbras, $2,3,9$

$$
\begin{aligned}
\boldsymbol{u}^{\alpha(\mathrm{PTD})}(\boldsymbol{x})= & \boldsymbol{u}^{\alpha(\mathrm{Kir})}(\boldsymbol{x})+\boldsymbol{u}^{\alpha(\mathrm{Ray})}(\boldsymbol{x}) \\
& +\sum_{\beta=\mathrm{L}, \mathrm{TV}, \mathrm{TH}} \boldsymbol{D}_{\beta}^{\alpha(\mathrm{Corr})}\left(\theta_{\beta}, \phi^{\alpha}, \theta^{\alpha}\right) \frac{\mathrm{e}^{i k_{L}\left[\zeta^{\alpha} \cdot x_{\beta}+\kappa_{\beta} R_{\beta}\right]}}{\left(k_{\beta} R_{\beta}\right)^{1 / 2}},
\end{aligned}
$$

where the smooth GTD correction to the Kirchhoff diffraction coefficient is

$$
D_{\beta}^{\alpha(\mathrm{Corr})}=D_{\beta}^{\alpha(\mathrm{GTD})}-D_{\beta}^{\alpha(\mathrm{Kir})} .
$$

\section{A NOVEL NUMERICAL SCHEME FOR THE CANONICAL EDGE DIFFRACTION PROBLEM}

Only the scattered $T$ fields are considered below, since only they involve the head waves, the main focus of this paper. In order to alleviate comparison of such description with the far-field asymptotics, each scattered $T(T V$ or $T H)$ field [Eq. (5)] is represented as

$$
\boldsymbol{u}_{T}^{\alpha(\mathrm{sc})}(\boldsymbol{x})=\boldsymbol{S}_{T}^{\alpha}\left(\theta_{T}, \phi^{\alpha}, \theta^{\alpha}, a_{T}\right) \frac{\mathrm{e}^{i k_{L}\left(\zeta^{\alpha} \cdot x_{T}+\kappa_{T} R_{T}\right)}}{\left(k_{T} R_{T}\right)^{1 / 2}},
$$

where using Eq. (6), each vector scattering coefficient $S$ $=S \boldsymbol{d}$ has the magnitude

$$
\begin{aligned}
S_{T}^{\alpha}\left(\theta_{T}, \phi^{\alpha}, \theta^{\alpha}, a_{T}\right) \\
=-i\left(k_{T} R_{T}\right)^{1 / 2} \int_{-\infty}^{\infty} \frac{\left[\boldsymbol{v}_{T}(\boldsymbol{\zeta}) \cdot \boldsymbol{v}^{\alpha}\left(\boldsymbol{\zeta}^{\alpha}\right)\right]\left[\boldsymbol{d}_{T}(\boldsymbol{\zeta}) \cdot \boldsymbol{d}_{T}\left(\boldsymbol{\zeta}_{T}\right)\right]}{\gamma_{T}\left(\xi_{1}\right)\left(\xi_{1}+\xi_{1}^{\alpha}\right)} \\
\quad \times \mathrm{e}^{i a_{T} f_{T}\left(\xi_{1}\right)} d \xi_{1}
\end{aligned}
$$

with the angle $\theta_{T}^{\mathrm{GE}}$ defined by the Snell law $\kappa_{T} \xi_{T, 1}^{\mathrm{GE}}=\kappa^{\alpha} \xi_{1}^{\mathrm{in}}$; $a_{T}=k_{T} R_{T} / 2 \pi$-the non-dimensionalized distance from the diffraction to observation point; and phase function

$$
\begin{aligned}
f_{T}\left(\xi_{1}\right) & =\frac{k_{L}}{2 \pi a_{T}}\left(\boldsymbol{\zeta}^{\alpha} \cdot \boldsymbol{x}_{T}+\kappa_{T} R_{T}\right) \\
& =\kappa_{T}^{-2}\left[-\xi_{1} \xi_{T, 1}+\gamma_{T}\left(\xi_{1}\right)\left|\xi_{T, 3}\right|-\gamma_{T}^{2}(0)\right] .
\end{aligned}
$$

Similarly, Eq. (8) can be used to introduce the scalar Kirchhoff scattering coefficient

$$
\begin{aligned}
S_{T}^{\alpha(\mathrm{Kir})} & \left(\theta_{T}, \phi^{\alpha}, \theta^{\alpha}, a_{T}\right) \\
= & -2 \pi i\left(k_{T} R_{T}\right)^{1 / 2} \operatorname{Res}_{T}\left(-\xi_{1}^{\alpha}\right) H\left(\cos \theta_{T}^{\mathrm{GE}}-\cos \theta_{T}\right) \\
& -i\left(k_{T} R_{T}\right)^{1 / 2}\left[\mathbf{v}_{T}\left(\zeta_{T}^{\mathrm{GE}}\right) \cdot \boldsymbol{v}^{\alpha}\left(\zeta^{\alpha}\right)\right]\left[\boldsymbol{d}_{T}\left(\zeta_{T}^{\mathrm{GE}}\right) \cdot \mathbf{d}_{T}\left(\zeta_{T}\right)\right] \\
& \times \int_{-\infty}^{\infty} \frac{1}{\gamma_{T}\left(\xi_{1}\right)\left(\xi_{1}+\xi_{1}^{\alpha}\right)} \mathrm{e}^{i a_{T} f_{T}\left(\xi_{1}\right)} d \xi_{1}
\end{aligned}
$$

and PTD correction to the scalar Kirchhoff scattering coefficient

$$
S_{T}^{\alpha(\text { Corr })}=S_{T}^{\alpha}-S_{T}^{\alpha(\text { Kir })} .
$$

Let us denote the integrals in Eqs. (16) and (18) by $\mathcal{I}_{\infty}$ to indicate that the integration is over the entire real axis. Note that outside the interval $I=\left[-\gamma_{T}(0), \gamma_{T}(0)\right]$ the magnitude of their integrands is exponentially decaying. In most cases of interest, $I$ contains the stationary point $\xi_{s t}=-\xi_{T, 1}$ and GE pole $\xi_{p}$ and inside $I$, the integrand is rapidly oscillating. The endpoints of this interval are weak singularities $\left(\lim _{\xi_{1} \rightarrow \pm \gamma_{T}(0)}\left|\gamma_{T}\left(\xi_{1}\right)\right|=0\right.$ ) as well as accumulation points [so that as they are approached from within, the speed of integrand oscillation increases without bounds, $\lim _{\xi_{1} \rightarrow \pm \gamma_{T}(0) \mp 0}$ $\left|f_{T}^{\prime}\left(\xi_{1}\right)\right|=\infty$-see Fig. 3(a)]. When $\left|\xi_{2}\right| \leq 1, I$ in Eq. (16) contains the branch point $\xi_{b r}=-\gamma_{L}(0)$ too. There are numerical schemes, which handle combinations of the challenges presented by the above features, ${ }^{22,23}$ but as far as we know our scheme is the first one to address all of them at once, striking a reasonable trade-off between accuracy and speed. Below we describe how. 
(a)

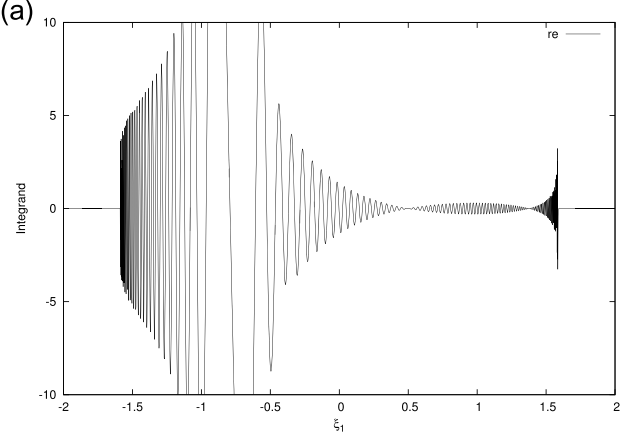

(c)

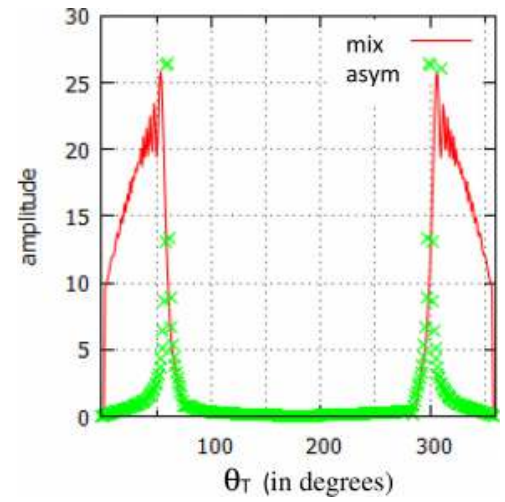

(b)

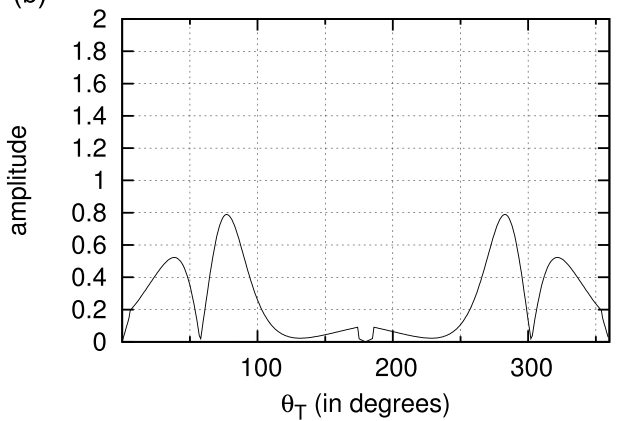

(d)

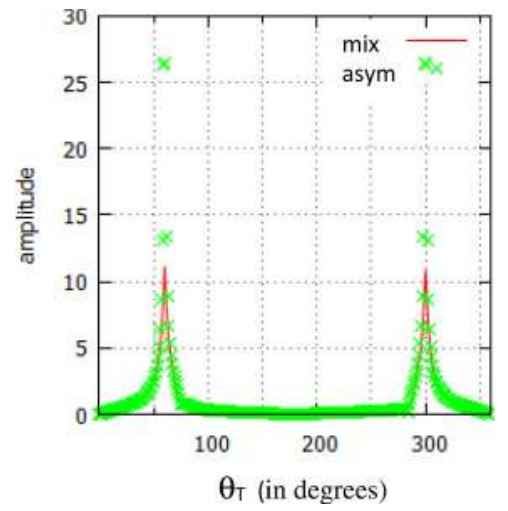

FIG. 3. (Color online) Testing the numerical code for $\phi^{T}=\theta^{T}=60^{\circ}$ $\left(\theta_{\mathrm{H}}=77^{\circ}\right)$ and $a_{T}=80$ : (a) Real part of typical integrand in Eq. (16): $\theta=$ $60^{\circ}, I \approx[-1.589,1.589], I_{\delta} \approx[-1.513$, 1.387], $\quad \xi_{b r} \approx-0.398, \quad \xi_{s t}=\xi_{p}$ $\approx-0.795$; (b) $\Delta S_{T V}^{T V \text { (mod) }}$ [Eq. (25)]; (c) $S_{T V}^{T V}$ [num, Eq. (16)] vs $D_{T V}^{T V}$ [asym, Eq. (10)]; (d) $S_{T V}^{T V}$ [num, Eq. (24)] vs $D_{T V}^{T V}$ [asym, Eq. (10)].

\section{A. The integration interval}

Even at high frequencies, truncating the integration interval to $I$ is not helpful, because $\pm \gamma_{T}(0)$ are accumulation points. Instead it is truncated to $I_{\delta}=\left[\xi_{1}^{-}, \xi_{1}^{+}\right]$, where $\xi_{1}^{ \pm}= \pm \gamma_{T}(0) \mp \delta_{ \pm}$. To assure that both $\xi_{s t}$ and $\xi_{b r}$ lie inside $I_{\delta}$, the offsets are chosen to be $\delta_{ \pm}=\delta \min \left(\left|\gamma_{T}(0) \mp \xi_{s t}\right|\right.$, $\left.\left|\gamma_{T}(0) \mp \xi_{b r}\right|\right)$. It has been established by trial and error that the optimal choice of $\delta$ is 0.1 : When $\delta<0.1$ the results are the same but the code takes longer to run; when $\delta>0.1$ the quality of results is inferior.

To compensate for the truncation of the integration interval from $(-\infty, \infty)$ to $I_{\delta}$ the integrand in $\mathcal{I}_{\infty}$ is represented as $F_{1} \exp \left(i a_{T} f_{T}\right)$ and the contribution of its semiinfinite tails is estimated integrating by parts and neglecting terms of order $O\left(a_{T}^{-2}\right)^{3}$

$$
\begin{aligned}
& \mathcal{I}_{\infty} \approx \mathcal{I}_{\delta}-\left.\left[\frac{1}{i a_{T} f_{T}^{\prime}\left(\xi_{1}\right)} F_{1}\left(\xi_{1}\right) \mathrm{e}^{i a_{T} f_{T}\left(\xi_{1}\right)}\right]\right|_{\xi_{1}^{-}} ^{\xi_{1}^{+}}, \\
& \mathcal{I}_{\delta}=\int_{\xi_{1}^{-}}^{\xi_{1}^{+}} F_{1}\left(\xi_{1}\right) \mathrm{e}^{i a_{T} f_{T}\left(\xi_{1}\right)} d \xi_{1} .
\end{aligned}
$$

The subtracted term above is referred to as the Borovikov correction.

\section{B. Accumulation points and weak singularities}

In the presence of accumulation points, choosing the nodes is an issue and instead of the standard Simpson's rule a composite Simpson's rule is implemented, briefly reported before. $^{24}$ This involves partitioning $I_{\delta}$ both sides of $\xi_{s t}$ (whose neighborhood makes the largest contribution) a number of times, each time halving the two subintervals closest to the endpoints of $I_{\delta}$. We denote the resulting subintervals by $I_{m}^{ \pm}=\left[\xi_{m}^{-}, \xi_{m}^{+}\right], m=1, \ldots, M$, with the $+/-$ superscript indicating that the subinterval lies to the right/left of $\xi_{s t}$. For each resulting subinterval, the minimum number of nodes $n_{m}$ is estimated by dividing its length by the length of its smallest integrand cycle $\ell_{m}=2 \pi / k_{T} \mid f_{T}^{\prime}\left(\xi_{m}^{ \pm} \mid\right.$, and this upper bound on the number of cycles is multiplied by 10 , to cover each cycle fully. If an integration subinterval is close to an accumulation point (its length is $<0.05) n_{m}$ is scaled by $1 / \gamma_{T}\left(\xi_{m}^{ \pm}\right)$-it has been checked that this accounts for the weak singularities at $\pm \gamma_{T}(0)$ as well as the GaussChebyshev scheme does. On each subinterval the standard Simpson's rule is applied. The optimal number of subintervals $M$ has been established by trial and error to be 22: For $M>22$ there is no improvement in the run-time or quality of results; for $M<22$ the run-time is greater.

\section{Poles}

Let $I$ contain $\xi_{p}$. If this pole lies outside $I_{\delta}$ the corresponding endpoint of $I_{\delta}$ is moved to $\xi_{1}^{ \pm}= \pm \gamma_{T}(0)$ $\mp 0.001 \delta\left(\gamma_{T}(0) \mp \xi_{p}\right)$, so that $\xi_{p}$ is absorbed into the redefined $I_{\delta}$. If $\xi_{p}$ lies too close to an accumulation point $\left(\gamma_{T}(0) \mp \xi_{p}<10^{-10}\right)$ but far from the stationary point $\left(k_{T}\left[f_{T}\left(\xi_{p}\right)-f_{T}\left(\xi_{s} t\right)\right]<\pi\right)$, its contribution is negligible and $I_{\delta}$ is not redefined.

When $I_{\delta}$ contains a pole, we introduce $F_{2}\left(\xi_{1}\right)=$ $F_{1}\left(\xi_{1}\right)\left(\xi_{1}-\xi_{p}\right) \exp \left[i a_{T} f_{T}\left(\xi_{1}\right)\right]$ to write ${ }^{1}$

$$
\mathcal{I}_{\delta}=\int_{\xi_{1}^{-}}^{\xi_{1}^{+}} \frac{F_{2}\left(\xi_{1}\right)}{\xi_{1}-\xi_{p}} d \xi_{1}=\left[p \cdot v \cdot \int_{\xi_{1}^{-}}^{\xi_{1}^{+}}+\int_{C}\right] \frac{F_{2}\left(\xi_{1}\right)}{\xi_{1}-\xi_{p}} d \xi_{1},
$$


where $C$ is a small half-circle passing under the pole $-\xi_{1}^{\alpha}$, so that the second term above is $\pi i F_{2}\left(-\xi_{1}^{\alpha}\right)$, half the standard pole contribution. We also re-write the Cauchy Principal Value integral as

$$
\begin{aligned}
p . v . \int_{\xi_{1}^{-}}^{\xi_{1}^{+}} \frac{F_{2}\left(\xi_{1}\right)}{\xi_{1}-\xi_{p}} d \xi_{1}= & \int_{\xi_{1}^{-}}^{\xi_{1}^{+}} \frac{F_{2}\left(\xi_{1}\right)-F_{2}\left(\xi_{p}\right)}{\xi_{1}-\xi_{p}} d \xi_{1} \\
& +F_{2}\left(\xi_{p}\right) \cdot \ln \left(\frac{\xi_{1}^{+}-\xi_{p}}{\xi_{p}-\xi_{1}^{-}}\right) .
\end{aligned}
$$

This assures a numerically stable evaluation, unless the pole is located at an interior node $\xi_{j}$ or else at either endpoint $\xi_{1}^{ \pm}$. To avoid this eventuality $I_{\delta}$ is first partitioned to establish what node shift would assure that the pole is half-way between two neighboring nodes, then the endpoints of $I_{\delta}$ are shifted accordingly and the new $I_{\delta}$ is repartitioned as above.

Note that the Rayleigh pole lies outside $I$ and therefore its contribution is evaluated in a standard manner using the Cauchy Residue Theorem.

\section{Removal of asymptotic pole contribution (optional)}

In the vicinity of pole $\xi_{p}$ the phase in $\mathcal{I}_{\delta}$ is

$$
f_{T}\left(\xi_{1}\right) \approx f_{T}\left(\xi_{p}\right)+f_{T}^{\prime}\left(\xi_{p}\right)\left(\xi_{1}-\xi_{p}\right)
$$

In the high-frequency regime the asymptotic contribution of regular integrand points is negligible and therefore the interval of integration can be extended beyond the limits of validity of Eq. (23) to $(-\infty, \infty)$. To satisfy the radiation condition, the resulting integral can be evaluated by closing the integration contour in the upper $\xi_{1}$ half-plane. When $f_{T}^{\prime}\left(\xi_{p}\right)<0$ the Jordan Lemma is inapplicable and the pole makes no contribution. It can be easily checked that the stationary point is a maximum and hence $f_{T}^{\prime}\left(\xi_{p}\right)>0$ when $\xi_{p}<\xi_{s t}$. Then the Jordan Lemma is applicable and the pole's asymptotic contribution ${ }^{1}$ describes the outgoing reflected or compensating wave. If only diffraction is of interest this contribution can be subtracted to modify $\mathcal{I}_{\delta}$ in Eq. (21) to

$$
\begin{aligned}
\mathcal{I}_{\delta}^{d}= & p \cdot v \cdot \int_{\xi_{1}^{-}}^{\xi_{1}^{+}} \frac{F_{2}\left(\xi_{1}\right)}{\xi_{1}-\xi_{p}} d \xi_{1} \\
& +\left[-\frac{1}{2}+H\left(-\operatorname{sgn} f_{T}^{\prime}\left(\xi_{p}\right)\right)\right] 2 \pi i F_{2}\left(\xi_{p}\right) .
\end{aligned}
$$

\section{E. Branch cut}

The third component of $\boldsymbol{v}^{T}\left(\xi_{1}, x_{2}, x_{3}\right)$ in Eq. (6) contains the factor $\gamma_{L}\left(\xi_{1}\right)$. Since the radiation condition requires the integration contour to encircle the upper half-plane (see Sec. VD), when $\left|\xi_{2}\right|>1(\leq 1)$ it is natural to make the branch cut from the imaginary (real) $\xi_{b r}=-\gamma_{L}(0)$ down (left), ${ }^{21}$ and when $\xi_{b r}$ is real to run the integration contour along the upper side of the cut. Then the cut makes no contribution when $\xi_{b r}$ is imaginary-for obvious reasons (in physical terms, the incidence is subcritical) and also when $\xi_{b r}$ is real, isolated and such that $f_{T}^{\prime}\left(\xi_{b r}\right) \geq 0$ - as a consequence of the Jordan Lemma and Cauchy Residue Theorem-since the integrand is holomorphic in the upper half-plane and exponentially decreasing along the positive part of the imaginary axis (in physical terms, we are in the shadow of the head wave.) The same reasoning applies when $f_{T}^{\prime}\left(\xi_{b r}\right)=0$ (that is, when $\left.\xi_{b r}=\xi_{s t}\right)$, because $\xi_{s t}$ is a maximum and therefore $f_{T}^{\prime \prime}\left(\xi_{s t}\right)<0$. When $f_{T}^{\prime}\left(\xi_{b r}\right)<0$ the Jordan Lemma is inapplicable and the contribution of the real branch cut is non-zero (in physical terms, we are in the region irradiated by the head wave.)

\section{F. Limits of applicability of the proposed scheme}

Due to the asymptotic nature of the Borovikov correction, similar to a recent numerical algorithm, ${ }^{23}$ but in contrast to many standard schemes (see references therein), as the non-dimensionalized distance increases so does the quality of the scheme. It fails at near grazing incidence angles when $\xi_{2} \approx \kappa^{2}$ [since then $\gamma_{T}(0) \approx 0$ and hence $I_{\delta} \approx 0$ ], which can be handled by calculating the total field rather than the scattered field. ${ }^{2}$ The scheme also fails at near grazing scattering angles (when near the crack boundary the diffracted waves interfere with the Rayleigh wave, the Rayleigh pole lying near an accumulation point). However, at grazing scattering angles the branch point can be near the stationary point only if the incidence is grazing. If not, at high frequencies the classical GTD applies.

\section{G. Mixed GTD/Simpson's scheme}

To speed up the code, the above numerical scheme for calculating the scattering coefficient [Eq. (24)] should be used in the critical zones only, otherwise relying on GTD or PTD. A critical zone where a head wave interferes with an edge diffracted wave is defined by the proximity condition $2 \pi a_{T}\left|f_{T}\left(\xi_{s t}\right)-f_{T}\left(\xi_{b r}\right)\right|<6 \pi$, with the coefficient 6 chosen to assure that the diffraction coefficients are smooth functions of $\theta_{T}$. A similar definition describes proximity of a branch point to pole. The resulting estimates are denoted by $\bar{S}$.

\section{CROSS-VALIDATION OF THE PROPOSED NUMERICAL SCHEME AND GTD AND PTD}

In all numerical examples reported below, $c_{L}=$ $5890 \mathrm{~m} \mathrm{~s}^{-1}$ and $c_{T}=3210 \mathrm{~m} \mathrm{~s}^{-1}$ as in mild steel. For easy comparison with the previously reported diffraction coefficients $^{20}$ the $T V-T H$ decomposition was defined using the incidence plane.

The treatment of the integration interval and pole proposed in Sec. V C, has been tested by evaluating [cf. Eq. (24)]

$$
\begin{aligned}
\Delta \mathcal{I}_{\delta}^{(\mathrm{mod})}= & p \cdot v \cdot \int_{\xi_{1}^{-}}^{\xi_{1}^{+}} \frac{F_{2}\left(\xi_{1}\right)}{\xi_{1}-\xi_{p}} \mathrm{e}^{-\left(2\left(\xi_{1}-\xi_{p}\right)^{2} / d^{2}\right)} d \xi_{1} \\
& +\left[-\frac{1}{2}+F\left(-\operatorname{sgn} f_{T}^{\prime}\left(\xi_{p}\right), \operatorname{sgn}\left(f_{T}^{\prime \prime}\left(\xi_{s t}\right)\right)\right]\right. \\
& \times 2 \pi i F_{2}\left(-\xi_{p}\right),
\end{aligned}
$$

where $d=\min \left(\xi_{p}+\gamma_{T}(0)-\delta, \gamma_{T}(0)-\delta-\xi_{p}\right) ;$ and the Fresnel functions are defined as 


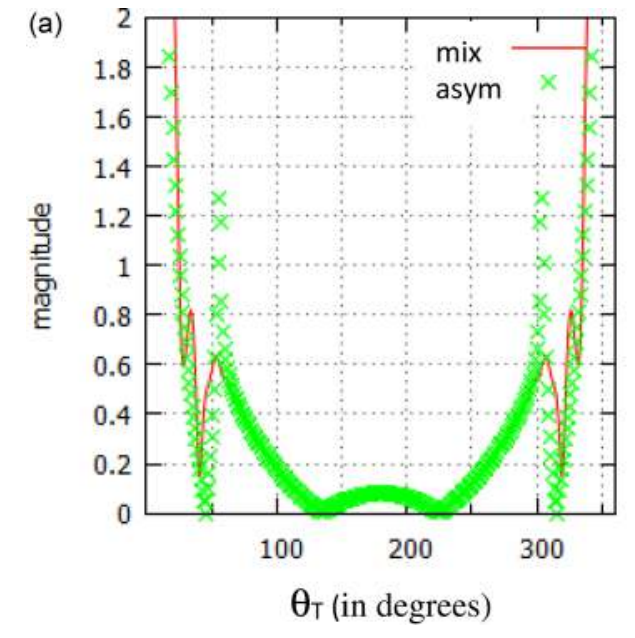

$$
F(x, \pm)=\frac{1}{\sqrt{\pi}} \int_{-\infty}^{x} e^{ \pm i\left(s^{2}-\pi / 4\right)} d s
$$

The Gaussian exponential factor has been introduced above to assure that the modified integrand is analytic. The factor 2 in its exponent has been chosen by trial and error to assure a trade-off between the requirements that the whole vicinity of $\xi_{s t}$ is covered and that at the endpoints $\xi_{1}^{ \pm}$of the modified integrand is small. Since the integrand is analytic and almost has a finite support on the integration interval, the Fresnel term in Eq. (25) estimates the uniform asymptotic pole contribution to the integral there ${ }^{1}$ better than the non-uniform Heaviside term in Eq. (24). Since the finite support condition is not satisfied exactly the asymptotics are not rigorous and the error [Eq. (25)] is not purely numerical. At the observation point where the above trade-off is optimal (this happens to be $\theta=58.5^{\circ}$ ) — see Fig. 3(b) — the corresponding relative error is $0.05 \%$.

The treatment of the integration interval proposed in Sec. V has been tested further against GTD and the highfrequency PTD, because when well within their limits of validity, their accuracy had been well established. Figures 3(c) and 3(d) demonstrate the effect of subtracting the asymptotic pole contribution in Eq. (24): In Fig. 3(c) this contribution describes for $\theta^{T}<60^{\circ}$ the compensating field and for $\theta^{T}>300^{\circ}$, the reflected field. The
FIG. 4. (Color online) $\bar{S}_{T V}^{T V}$ [mix, Eq. (16), from now on calculated using the mixed GTD/Simpson's scheme] vs $D_{T V}^{T V}$ [asym, Eq. (10)], $\phi^{T}=90^{\circ}, \theta^{T}=$ $0^{\circ}\left(\theta_{\mathrm{H}}=57^{\circ}\right): a_{T}=$ (a) 10 ; (b) 500 .

\section{$\theta_{\mathrm{T}}$ (in degrees)}

contribution grows with distance, since it contains the factor $\left(k_{T} R_{T}\right)^{1 / 2}$ and the plane waves have constant amplitudes. In Fig. 3(d) only the pure edge diffracted field in the irradiated regions and interference patterns in the penumbras are present. The figure shows that outside critical regions the proposed numerical scheme produces the same results as GTD.

Let us now apply the new scheme for description of the critical zones where the head waves interfere with edge diffracted waves only. Typical $T V-T V$ scattering coefficients $\bar{S}$ [Eq. (16)] and GTD diffraction coefficients $D$ [Eq. (10)] are presented in Figs. 4 and 5. It is clear that at smaller $a_{T}$ the critical spikes in scattering coefficients are smoothed. At larger distances, somewhat surprisingly, they turn out to be the same as the critical spikes in GTD diffraction coefficients. Numerical experiments confirm that they do not grow beyond $a_{T}=500$. Thus, the critical spikes in GTD diffraction coefficients are physical in nature. They must be due to the energy redistribution inside the critical zones, with practically no edge diffracted $T$ waves propagating through the regions, which do not support the head waves. ${ }^{25}$ The smoothing near the edge must be due to destructive interference between head waves and diffracted waves, ceasing away from the edge, because around critical rays the uniform asymptotics of Eq. (15) decay faster ${ }^{1}$ than the stationary phase asymptotics [Eq. (9)].

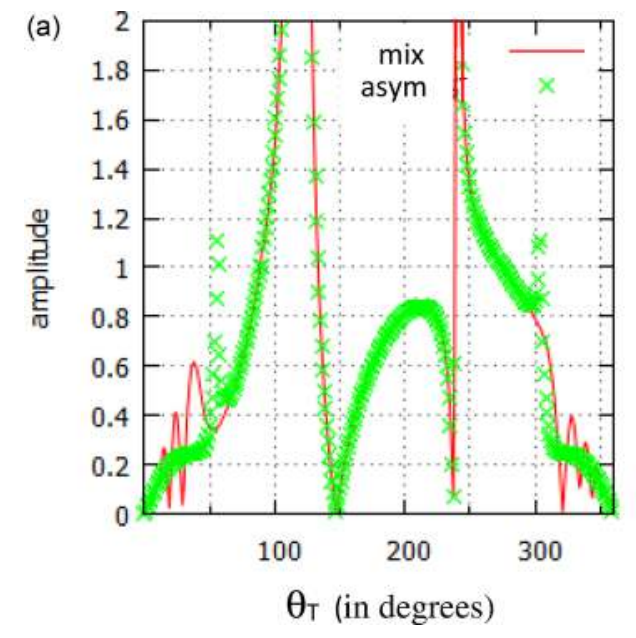

FIG. 5. (Color online) $\bar{S}_{T V}^{T V}$ vs $D_{T V}^{T V}$. $\phi^{T}=90^{\circ}, \theta^{T}=120^{\circ}\left(\theta_{\mathrm{H}}=57^{\circ}\right) . a_{T}$ ' $\mathrm{s}$ and key as in Fig. 4. 


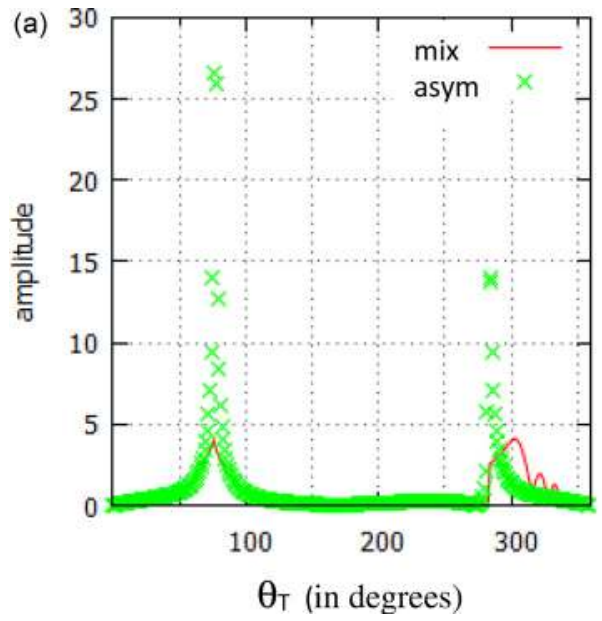

Let us turn to the critical zones where the head waves interfere with both edge diffracted and GE waves. The typical $T V$ - $T V$ scattering coefficients $\bar{S}$ [Eq. (16)] and GTD diffraction coefficients $D$ [Eq. (10)] are presented in Fig. 6. The figure shows that as above, in critical regions, at smaller $a_{T}$ the numerical scheme produces the scattering coefficients smaller than GTD, while the critical spikes grow even for $a_{T} \gg 500$.

Moving from GTD to PTD, typical corrections to scattering [Eq. (19)] and corrections to diffraction coefficients [Eq. (14)] are presented in Fig. 7. At $a_{T}=500$ the critical spikes are the same whether calculated using Eq. (14) or Eq. (19). Beyond $a_{T}=500$ the critical spikes in $S^{\text {Corr }}$ do not grow, confirming the physical nature of such spikes in $D^{\text {Corr }}$.

To conclude, not only have we tested the new scheme against GTD and high-frequency PTD, we performed cross- validation as well: There has been a general consensus that GTD does not apply near the critical angles, but we have shown that in the very far field it does.

\section{A NOVEL SCHEDULE FOR DESCRIBING CRITICAL ZONES IN THE DIFFRACTED FIELD}

Whether a critical zone overlaps a penumbra or not the distance dependence of the scattered field $\boldsymbol{u}_{T}^{\alpha(\mathrm{sc})}$ has been shown ${ }^{1,12}$ to be roughly $a_{T}^{-3 / 4}$, so that in critical zones the difference between the respective correction coefficients [Eqs. (19) and (14)] has to be decreasing as $a_{T}^{-1 / 4}$. Typical plots are presented in Figs. 8 and 9. It follows that in the critical regions the scattered field $\boldsymbol{u}_{T}^{T \text { (crit) }}$ can be modeled using a relatively fast semi-analytical schedule

$$
\boldsymbol{u}_{T}^{T(\text { crit })}(\boldsymbol{x})=\boldsymbol{u}_{T}^{T(\text { Kir })}(\boldsymbol{x})+\frac{\mathrm{e}^{i k_{L}\left(\zeta^{\alpha} \cdot \boldsymbol{x}_{T}+\kappa_{T} R_{T}\right)}}{\left(k_{T} R_{T}\right)^{1 / 2}} \cdot \begin{cases}\boldsymbol{S}_{T}^{\alpha(\text { Corr })}, & a_{T}<a_{T(\min )}, \\ \boldsymbol{D}_{T}^{\alpha(\text { Corr })}+\frac{\left[\boldsymbol{S}_{T}^{\alpha(\text { Corr })}-\boldsymbol{D}_{T}^{\alpha(\text { Corr })}\right]_{a_{T(\min )}},}{\left(a_{T} / a_{T(\min )}\right)^{1 / 4}} & \left.a_{T(\min )} \leq a_{T} \leq a_{T(\max )}\right) \\ \boldsymbol{D}_{T}^{\alpha(\text { Corr })}, & a_{T}>a_{T(\max )},\end{cases}
$$

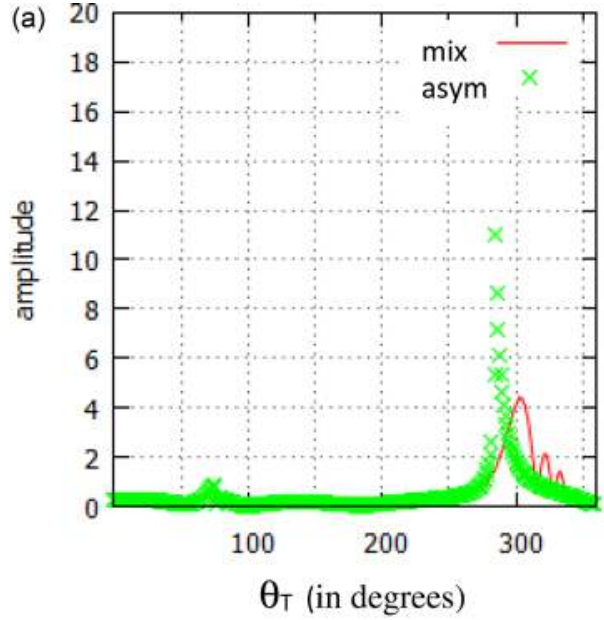

FIG. 7. (Color online) $\bar{S}_{T V}^{T V(\text { Corr })}$ [mix, Eq. (19)] vs $D_{T V}^{T V \text { (Corr) }}$ [asym, Eq. (14)]. Configurations as in Fig. 6. 

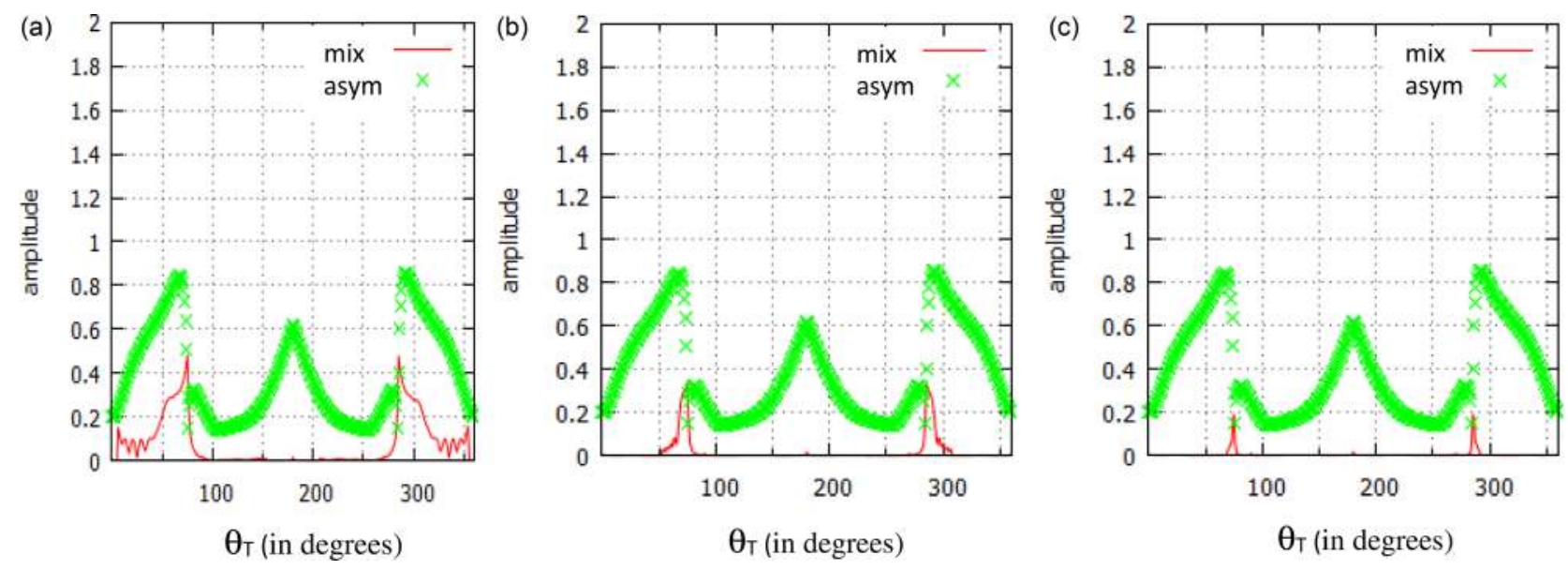

FIG. 8. (Color online) $\bar{S}_{T V}^{T V(\text { Corr })}-D_{T V}^{T V(\text { Corr })}$ (mix) vs $D_{T V}^{T V(\text { Corr })}$ [asym, Eq. (14)]. $\phi^{T}=60^{\circ}, \theta^{T}=120^{\circ},\left(\theta_{\mathrm{H}}=77.5^{\circ}\right): a_{T}=($ a) $10 ;($ b) $500 ;$ (c) 1000 .

where for clarity of presentation, the arguments of the diffraction and scattering coefficients have been omitted. According to numerical experiments, in steel this simple recipe works for isolated critical regions when $a_{\mathrm{T}(\min )}=10$ and for the critical regions overlapping penumbras when $a_{\mathrm{T}(\min )}=40$. In both cases a good choice for $a_{T(\max )}$ is 500 , making the jump experienced by the scattering coefficient at $a_{T}=a_{T(\max )}$ very small.

\section{CONCLUSIONS}

A schedule has been proposed for describing critical zones in the scattered field. It is based on a novel mixed GTD/Simpson's scheme for simulating the head waves and associated critical regions, whether the latter overlap penumbras or not and at any frequency and distance to diffracting edge. The numerical component of the scheme has been shown to have accuracy sufficient in envisaged applications. It implements fast integration over the real axis of the integrand, which has a branch point, stationary point, and maybe a pole inside a finite interval on this axis, is exponentially decreasing outside this interval while oscillating inside it so that the endpoints of the interval are the integrand's accumulation points.

It has been shown that in the very far field, when a critical region overlaps no penumbra, the classical GTD diffraction coefficients give a good description of edge diffracted waves and when such overlap takes place good results can be obtained with the high-frequency version of PTD. Though there is no mathematical justification for these asymptotics to be applicable when the rapidly oscillating integrand possesses a branch point coalescing with the stationary phase point or pole, the result is consistent with the fact that the head waves decay with distance faster than edge diffracted waves.

It is envisaged that the proposed schedule for simulating critical zones is implemented in CIVA [software platform developed at CEA/LIST and widely used in NDE (Refs. 7 and 8)] in order to improve simulation of the ultrasonic response from large cracks near critical angle. The schedule could prove to be of interest in other branches of physics and mathematics too, as long as the effects they describe are presented in an integral form, with integrands containing a branch point coalescing with a stationary phase point and maybe a pole.
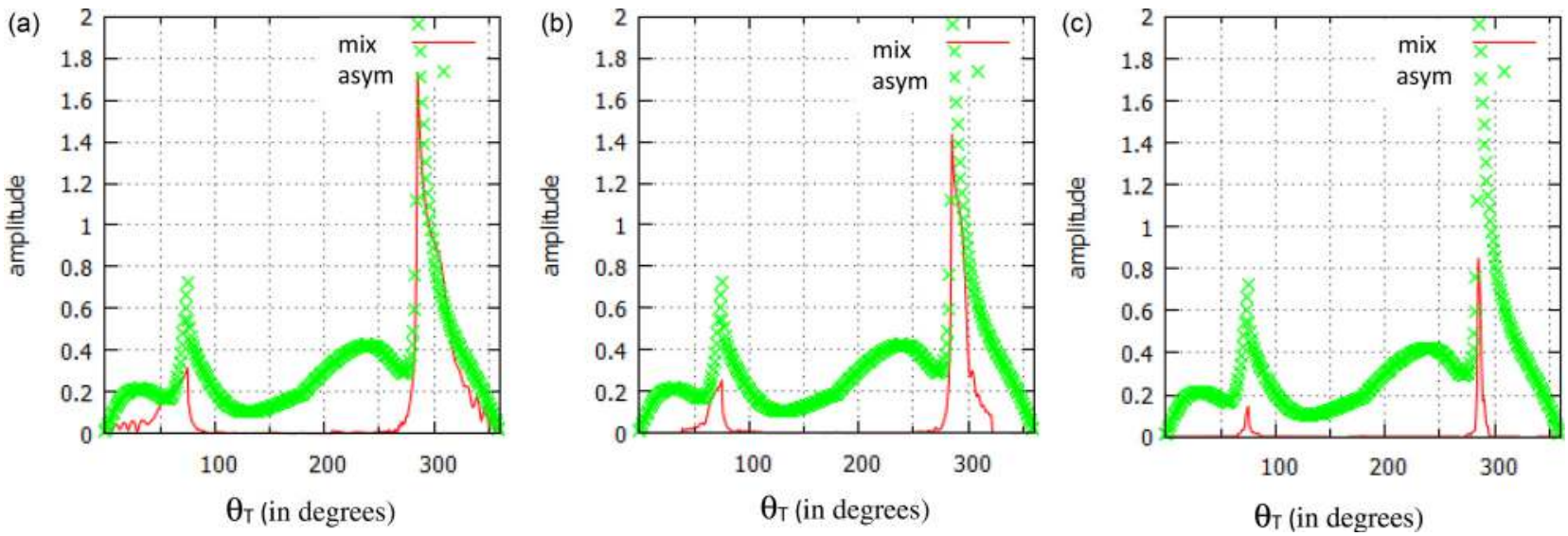

FIG. 9. (Color online) $\bar{S}_{T V}^{T V(\text { Corr })}-D_{T V}^{T V(\text { Corr) }}$ (mix) vs $D_{T V}^{T V(\text { Corr) }}$ [asym, Eq. (14)]. $\phi^{T}=60^{\circ}, \theta^{T}=90^{\circ}\left(\theta_{\mathrm{H}}=77.5^{\circ}\right): a_{T}=$ (a) 10 ; (b) 500 ; (c) 1000 . 


\section{ACKNOWLEDGMENTS}

This work has been carried out within the framework of the CIVAMONT 2020 collaborative project. Dr. M. Harmer contributed significantly to the numerical scheme. We are grateful to anonymous reviewers for many helpful suggestions.

${ }^{1}$ V. A. Borovikov, Uniform Stationary Phase Method (IEE, London, 1994), Secs. 1.6 and 2.4 .

${ }^{2} \mathrm{P}$. Y. Ufimtsev, Fundamentals of the Physical Theory of Diffraction (John Wiley and Sons, Inc., New York, 2007), Chaps. 1, 3, and 9.

${ }^{3}$ V. A. Borovikov and B. Y. Kinber, Geometrical Theory of Diffraction (IEE, London, 1994), Chap. 6, Sec. 3.7.

${ }^{4}$ J. B. Keller, "Geometrical theory of diffraction," J. Opt. Soc. Am. 52(2), 116-130 (1962).

${ }^{5}$ J. D. Achenbach, A. K. Gautesen, and H. McMaken, Ray Methods for Waves in Elastic Solids, With Applications to Scattering by Cracks (Pitman, New York, 1979), Sec. 5.3, pp. 1-251.

${ }^{6}$ G. Huet, M. Darmon, A. Lhémery, and S. Mahaut, "Modelling of corner echo ultrasonic inspection with bulk and creeping waves," in Ultrasonic Wave Propagation in Non Homogeneous Media, Springer Proceedings in Physics, Vol. 128 (Springer, Berlin, 2009), pp. 217-226.

${ }^{7}$ S. Mahaut, M. Darmon, S. Chatillon, F. Jenson, and P. Calmon, "Recent advances and current trends of ultrasonic modelling in CIVA," Insight 51(2), 78-81 (2009).

${ }^{8} \mathrm{M}$. Darmon and S. Chatillon, "Main features of a complete ultrasonic measurement model," Open J. Acoust. 3(3A), 43-53 (2013).

${ }^{9}$ V. Zernov, L. Fradkin, and M. Darmon, "A refinement of the Kirchhoff approximation to the scattered elastic fields," Ultrasonics 52(7), 830-835 (2011).

${ }^{10}$ A. Mohorovičić, "Das Beben vom 8. Oktober 1909," Jahrb. Meteorol. Obs. Zagreb Für 1909 4, 1-67 (1910). A translation appears in Geofizika 9, 3-55 (1992).

${ }^{11}$ V. Cerveny and R. Ravindra, Theory of Seismic Head Waves (University of Toronto Press, Toronto, 1971), pp. 1-312.
${ }^{12}$ W. S. Lihh, "Frequency- and time-domain asymptotic fields near the critical cone in fluid-fluid configuration," J. Acoust. Soc. Am. 121(5), 2655-2666 (2007).

${ }^{13}$ A. Ciarkowski, "Uniform asymptotic expansion of an integral with a saddle point, a pole and a branch point," Proc. R. Soc. London, Ser. A 426, 273-286 (1989).

${ }^{14}$ I. Thompson, “An improved uniform approximation for diffraction," Proc. R. Soc. London, Ser. A 462, 1341-1353 (2006).

${ }^{15} \mathrm{D}$. Gridin, "High-frequency asymptotic description of head waves and boundary layers surrounding the critical rays in an elastic half space," J. Acoust. Soc. Am. 104(3), 1188-1197 (1998).

${ }^{16}$ B. Caroli, C. Caroli, C. Misbah, and B. Roulet, "On velocity selection for needle crystals in the fully nonlocal model of solidification," J. Phys. 48, 547-552 (1987).

${ }^{17}$ R. Uitham and B. J. Hoenders, "The electromagnetic Brillouin precursor in one-dimensional photonic crystals," Optics Comm. 281, 5910-5918 (2008).

${ }^{18}$ M. V. Berry, "Causal wave propagation for relativistic massive particles: Physical asymptotics in action,” Eur. J. Phys. 33(2), 279-294 (2012).

${ }^{19}$ A. Kuznetsov, "On the Riemann-Siegel formula," Proc. R. Soc. London, Ser. A 463(2086), 2557-2568 (2007).

${ }^{20}$ J. D. Achenbach and A. K. Gautesen, "Edge diffraction in acoustics and elastodynamics (low and high frequency asymptotics)," in Low and High Frequency Asymptotics-Acoustic, Electromagnetic and Elastic Wave Scattering, edited by V. K. Varadan and V. V. Varadan, Vol. 2 (NorthHolland, Amsterdam, 1986), Chap. 4, pp. 335-401.

${ }^{21} \mathrm{~A}$. K. Gautesen, "A note on the diffraction coefficients for elastodynamic diffraction by sharp edges," Wave Motion 47(5), 327-332 (2010).

${ }^{22}$ L. N. Trefethen and J. A. C. Weideman, "The exponentially convergent trapezoidal rule," SIAM Rev. 56(3), 385-458 (2014).

${ }^{23}$ A. Iserles and S. P. Nørsett, "Efficient quadrature of highly oscillatory integrals using derivatives," Proc. R. Soc. London, Ser. A 461, 1383-1399 (2005).

${ }^{24}$ L. Fradkin, M. Harmer, and M. Darmon, "Edge diffraction coefficients around critical rays," in Proceedings of the 12th Anglo-French Physical Acoustics Conference, J. Phys.: Conf. Series 498, 012010 (2014).

${ }^{25}$ L. Ju. Fradkin and A. P. Kiselev, "The two-component representation of time-harmonic elastic body waves in the high- and intermediate-frequency regimes," J. Acoust. Soc. Am. 101(1), 52-65 (1997). 